\title{
Massive, Spontaneous Facial Hematoma in a Neurofibromatosis Type 1 Patient
}

\author{
Peter F. Svider ${ }^{1}$, Chirag R. Patel ${ }^{2}$, Sangeeta Lamba ${ }^{3}$, Chirag Gandhi ${ }^{1}$, Soly Baredes ${ }^{1}$, \\ Robert W. Jyung ${ }^{*}$ \\ ${ }^{1}$ Department of Otolaryngology_-Head \& Neck Surgery, University of Medicine and Dentistry of New Jersey, \\ New Jersey Medical School, Newark, USA \\ ${ }^{2}$ Department of Emergency Medicine, University of Medicine and Dentistry of New Jersey, \\ New Jersey Medical School, Newark, USA \\ ${ }^{3}$ Department of Neurological Surgery, University of Medicine and Dentistry of New Jersey, \\ New Jersey Medical School, Newark, USA \\ Email: *jyrungrw@umdnj.edu
}

Received November 19, 2012; received December 22, 2012; accepted January 2, 2013

\begin{abstract}
Significant hemorrhage in Neurofibromatosis Type 1 (NF-1) patients occurs infrequently, but has potentially devastateing consequences when occurring in the head and neck region. There have been no prior reports of patients with hemodynamically significant, rapidly-expanding lesions into a neurofibroma in the head and neck region without preceding trauma. This case describes the management of a spontaneous, rapidly expanding facial hematoma in an NF-1 patient with an extensive facial and skull base plexiform neurofibroma. The patient underwent angioembolization of his left external carotid artery prior to operative management. The strategies utilized can be extended to management of facial hematomas arising from more common situations such as fractures, lacerations, and pseudoaneursyms, along with bleeding from subacute conditions like other head and neck cancers.
\end{abstract}

Keywords: Neurofibromatosis Type 1; Facial Hematoma; Hemorrhage; Spontaneous Hemorrhage

\section{Introduction}

Significant hemorrhage in Neurofibromatosis Type 1 (NF-1) patients occurs infrequently, but has potentially devastating consequences when occurring in the head and neck region. There have been no prior reports of patients with hemodynamically significant, rapidly-expanding lesions into a neurofibroma in the head and neck region without preceding trauma. This case describes the management of a spontaneous, rapidly expanding facial hematoma in an NF-1 patient with an extensive facial and skull base plexiform neurofibroma. The patient underwent angioembolization of his left external carotid artery prior to operative management. The strategies utilized can be extended to management of facial hematomas arising from more common situations such as fractures, lacerations, and pseudoaneursyms, along with bleeding from subacute conditions like other head and neck cancers.

\section{Case Report}

A 50-year-old man with NF-1 presented to the emer-

*Corresponding author. gency department with sudden onset of progressive left facial swelling for 10 hours. His significant past medical history included resection of a left temporal area neurofibroma with reconstruction by the plastic surgery service 20 years prior. Following his resection, he had a barely noticeable left temporal subcutaneous neurofibroma and a $5 \mathrm{~cm}$ flat linear left temporo-facial scar (confirmed with a recent driver's license photograph) along with decreased visual acuity in the left eye secondary to a meningocele. He denied any recent trauma to the region but had noticed a "tight" feeling in the left temporal area for which he took ibuprofen prior to sleeping that night. He woke up due to pain and swelling in his left face.

On exam, the patient was awake, oriented, and afebrile with normal vital signs, resting comfortably with a massive left temporal, fluctuant swelling extending from his forehead and upper face to behind his left ear (Figure 1). He had a left facial scar along with multiple skin tags and macules over his trunk consistent with NF-1.

Diagnostic workup was significant for a hemoglobin of $8.4 \mathrm{~g} / \mathrm{dl}$ and the initial CT scan revealed a hyperdense soft tissue mass, extending superiorly to the left temperofrontal area, consistent with a hematoma (Figure 2). 
Notable chronic findings included dysplastic changes of the left sphenoid bone, ethmoid air cells, zygomatic arch, and dysplasia of the left orbital roof with an associated meningocele causing orbital enlargement and proptosis of the left globe. No intracranial hemorrhage was noted.

The hematoma showed signs of rapid growth, with tense overlying skin, diaphoresis, and punctuate hemorrhagic spots at the point of maximal stretch (Figure 3). After combined evaluation by the otolaryngology and endovascular services, it was decided that the optimal treatment would be angiography for identification and embolization of the responsible vessel(s), followed by evacuation of the hematoma.

Since no specific bleeder was identified during angiography, the external carotid artery was embolized. His

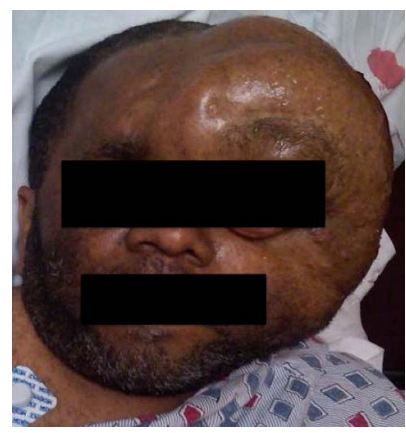

Figure 1. Initial presentation in the Emergency Department, approximately 2 hours after the onset of bleeding.

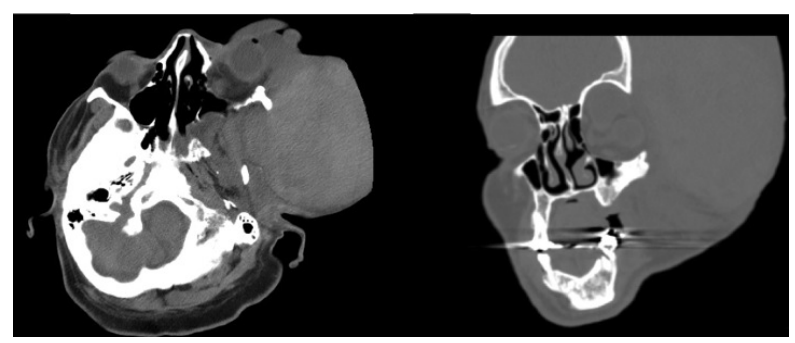

(a)

(b)

Figure 2. Computed Tomography Scan in the Emergency Department illustrating (a) axial and (b) coronal views of the left-sided hyperdense soft tissue mass, along with chronic dysplastic findings.

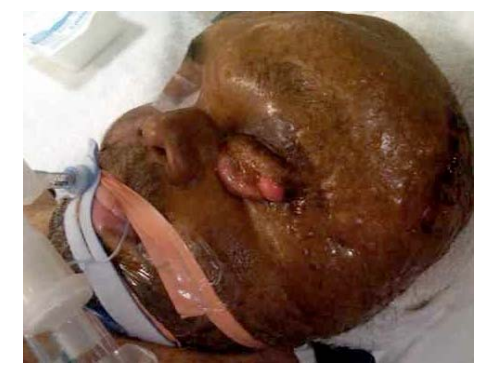

Figure 3. Rapidly growing hematoma with tense overlying skin and punctuate hemorrhages at the points of maximal stretch.

repeat hemoglobin at this time was $5 \mathrm{~g} / \mathrm{dl}$, and blood was transfused. Surgical exploration of the tense facial mass revealed copious amounts of clotted blood. Dissection revealed a diffuse neurofibroma with extremely friable blood vessels and persistent blood loss during repeated attempts at hemostasis. Bipolar and Bovie electrocautery were ineffective, and ligation was used to stop bleeding from the neurofibroma. By the end of the procedure, the estimated blood loss included 2.6 L in acute bleeding and 1.3 $\mathrm{L}$ of clotted blood evacuated, and the patient received 12 units packed red blood cells, 5 units FFP, and 10 units of platelets.

His post-operative course was complicated by anasarca and pulmonary edema secondary to fluid overload, along with difficulty weaning from the ventilator necessitating a tracheostomy on post-operative day 6. Healing of the facial wound was complicated by discharge of necrotic debris along with sloughing of the skin. On postoperative day 18, the patient was discharged to an acute rehabilitation facility and the mass and swelling on his face had subsided to a large extent but with continued sloughing and increased areas of apparent necrosis of the overlying facial skin.

On 1-month follow-up, the patient was doing well at his rehabilitation facility and his tracheostomy was decannulated in the clinic. Facial swelling had significantly subsided and was minimal, and no open facial wounds were present. Continued left-sided facial droop, excess skin, and laxity were present. As of 2 months post-discharge, the patient continues to do well and plans to continue follow-up for observation of the tumor, with future consideration of reconstructive options should the tumor remain stable.

\section{Discussion}

Neurofibromas are rarely associated with life-threatening hemorrhages, with even fewer cases of life-threatening rapidly expanding hematomas originating from facial lesions. To the best of our knowledge, this is the first such report of a rapidly expanding atraumatic facial hematoma from a neurofibroma. Timely recognition and management is crucial due to the risks of compression of airway, vascular and orbital structures.

NF1 is an autosomal dominant genetic disorder with a diverse range of phenotypic expression that can include multiple neurofibromas, optic gliomas, cafe au lait spots, and various other manifestations [1]. Depending on the extent and location of disease, functional and cosmetic considerations usually define decisions related to neurofibroma excision. Treatment strategies vary based on the predominant lesions and include management of complications such as malignant degeneration, mass effect on adjacent structures, and as in our case, the rare 
situation of significant hemorrhage.

Vascular abnormalities within and around neurofibromas contribute to their potential bleeding tendencies, ranging from vascular invasion by the tumors to arterial dysplasia within them. Most of the bleeding that occurs is clinically insignificant and goes unnoticed, with isolated cases of severe abdominal and thoracic cavity hemorrhage [2]. Following trauma, the abnormal vessel architecture can undergo a sequence where a vessel ruptures and subsequent bleeding increases local pressure on nearby vessels, causing them to rupture [3]. The friability of these abnormal vessels propagates this cycle, causing additional bleeding that is difficult to control, as demonstrated during our patient's procedure.

Hematologic abnormalities in NF1 patients have also been proposed as a possible cause of increased bleeding. An association with von Willebrand disease has been suggested, with possible platelet dysfunction demonstrated in vitro [4]. Additionally, spontaneous hemorrhage has been documented in a patient with truncal neurofibromas soon after starting anticoagulation [5]. However, neurofibroma-related hematomas are rare enough that it may be difficult to prove a significant association with platelet dysfunction.

The majority of spontaneous hemorrhages reported in NF1 are secondary to vascular plexiform variants of neurofibromas occurring in intrathoracic, retroperitoneal, and GI tract locations. Management traditionally involves aggressive hemodynamic resuscitation with crystalloids and blood products, along with surgical ligation of feeding vessels. There is one report of a scalp hematoma that utilized pre-operative embolization of the ipsilateral vertebral artery followed by definitive tumor resection two days later [6]. A similarly timed angiogram followed by definitive resection was not feasible in our patient. Since the initial CT scan was unable to distinguish between tumor and the hematoma, it was difficult to define the full extent of the tumor. In addition, there was evidence of intracranial communication with the orbit secondary to tumor erosion of the greater wing of the sphenoid.

There have been reported cases of non-spontaneous facial hematoma in NF1. One patient developed a rapidly expanding hematoma after sustaining trauma to the left temple and had pre-operative embolization of an intratumoral vessel followed by surgical ligation of the ipsilateral external carotid artery [7]. This case differs from ours not only in having a traumatic cause but also in that a specific vessel supplying the tumor was identified angiographically, allowing for more effective pre-operative embolization and a safer operation. The rate and size of our patient's hematoma rendered identifying specific feeder vessels impossible and the entire external carotid artery (minus two branches) had to be embolized preo- peratively before attempting open exploration.

While operative exploration is an appropriate method to evacuate a large hematoma in a critical location, endovascular treatment can be a valuable adjunct that stabilizes hematomas and decreases risks associated with open exploration in critical areas. Transarterial embolization of the external carotid artery (ECA) has been demonstrated to be safe and effective in decreasing bleeding in patients with other head and neck tumors. [8] Endovascular techniques have also been successfully used for vascular lesions resulting from acute facial trauma $[9,10]$. Angiographic embolization of the left external carotid artery was a conservative approach that stabilized the rapidly expanding hematoma, but it may have contributed to the necrosis and poor wound healing encountered post-operatively.

\section{Conclusion}

This case demonstrates how NF-1-derived tumors can lead to rapidly expanding hematomas in the head and neck, which are difficult to control and can have devastating consequences. Failure to promptly recognize and treat this condition can result in serious harm to surrounding structures, cause deficits in function and appearance, and potentially endanger a patient's airway. Angioembolization is a valuable adjunct to stabilize the bleeding, although operative intervention is ultimately needed with an expanding hematoma.

\section{REFERENCES}

[1] B. R. Korf, "Diagnosis and Management of Neurofibromatosis Type 1," Current Neurology and Neuroscience Reports, Vol. 1, No. 2, 2001, pp. 162-167. doi:10.1007/s11910-001-0012-z

[2] C. Nopajaroonsri and A. A. Lurie, "Venous Aneurysm, Arterial Dysplasia, and Near-Fatal Hemorrhages in Neurofibromatosis Type 1," Human Pathology, Vol. 27, No. 9, 1996, pp. 982-985. doi:10.1016/S0046-8177(96)90229-4

[3] T. C. Tung, Y. R. Chen, K. T. Chen, C. T. Chen and R. Bendor-Samuel, "Massive Intratumor Hemorrhage in Facial Plexiform Neurofibroma,” Head \& Neck, Vol. 19, No. 2, 1997, pp. 158-162. doi:10.1002/(SICI)1097-0347(199703)19:2<158::AID-H ED13>3.0.CO;2-9

[4] J. E. Rasko, K. N. North, E. J. Favaloro, L. Grispo and M. C. Berndt, "Attenuated Platelet Sensitivity to Collagen in Patients with Neurofibromatosis Type 1,” British Journal of Haematology, Vol. 89, No. 3, 1995, pp. 582-588. doi:10.1111/j.1365-2141.1995.tb08367.x

[5] L. Lessard, A. Izadpanah and H. B. Williams, "Giant Thoracic Neurofibromatosis Type 1 with Massive Intratumoral Haemorrhage: A Case Report,” Journal of Plastic, Reconstructive \& Aesthetic Surgery, Vol. 62, No. 9, 2009, pp. e325-e329. doi:10.1016/j.bjps.2007.10.071 
[6] M. Tsutsumi, K. Kazekawa, A. Tanaka, et al., "Rapid Expansion of Benign Scalp Neurofibroma Caused by Massive Intratumoral Hemorrhage-Case Report," Neurologia Medico-Chirurgica, Vol. 42, No. 8, 2002, pp. 338-340. doi:10.2176/nmc.42.338

[7] K. T. S. Kumakiri, "Neurofibromatosis with Massive Facial Hematoma," Otolaryngology_Head and Neck Surgery (Tokyo), Vol. 75, No. 7, 2003, pp. 473-476.

[8] Y. F. Chen, Y. C. Lo, W. C. Lin, et al., "Transarterial Embolization for Control of Bleeding in Patients with Head and Neck Cancer," Official Journal of American Academy of Otolaryngology_Head and Neck Surgery, Vol. 142, No.
1, 2010, pp. 90-94. doi:10.1016/j.otohns.2009.09.031

[9] Z. H. Baqain, C. Thakkar and N. Kalavrezos, "Superselective Embolization for Control of Facial Haemorrhage," Injury, Vol. 35, No. 4, 2004, pp. 435-438. doi:10.1016/j.injury.2003.09.001

[10] J. J. Borsa, A. B. Fontaine, J. M. Eskridge, J. K. Song, E. K. Hoffer and A. A. Aoki, "Transcatheter Arterial Embolization for Intractable Epistaxis Secondary to Gunshot Wounds," Journal of Vascular and Interventional Radiology: JVIR, Vol. 10, No. 3, 1999, pp. 297-302. doi:10.1016/S1051-0443(99)70034-5 